\title{
Existe-t-il réellement un nouveau paradigme de la chimie verte?
}

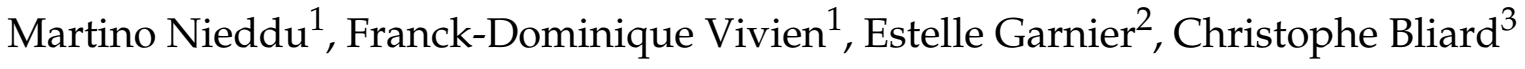 \\ 1 Économiste, Laboratoire REGARDS, Université de Reims Champagne Ardenne, 51096 Reims cedex, France \\ 2 Économiste, Responsable du département SHS à l'agence CARINNA ${ }^{1}, 51100$ Reims, France \\ 3 Chimiste, UMR 7312 CNRS, Institut de chimie moléculaire de Reims (ICMR), 51687 Reims cedex, France
}

\begin{abstract}
Science et industrie, la chimie présente des caractéristiques propres parmi les sciences. Après plusieurs décennies de fragmentations internes, et d'influences prépondérantes de la physique et de l'ingénierie industrielle, la discipline académique et le génie des procédés semblent rechercher inspirations et ressources dans les sciences et techniques du vivant. Ainsi, confrontés aux impasses environnementales (ressources et pollutions) des industries chimiques, aux conceptions technologiques qui les sous-tendent, et aux critiques et réactions fortes qu'elles suscitent, les chimistes, scientifiques et ingénieurs ont trouvé avec les douze principes de la "chimie verte » une charte de bonne conduite, déclinée dans les publications et manifestations qui ont marqué l'année internationale de la chimie (2011). Mais qu'en est-il en réalité ?
\end{abstract}

La Rédaction

\section{Mots-clés :}

chimie verte; rupture paradigmatique ; ressources agricoles ; voies technologiques ; patrimoine collectif
Résumé - La «chimie verte » est présentée par certains auteurs comme le nouveau paradigme de la chimie qui va lui permettre de répondre aux enjeux du développement durable. Nous discutons cette hypothèse en nous focalisant sur le domaine de l'usage des ressources agricoles pour la chimie (septième principe de la chimie verte). Après avoir rappelé la construction institutionnelle dont a fait l'objet la chimie verte, nous montrons que la chimie doit être appréhendée comme une discipline d'apprentissages orientés, les scientifiques cherchant à se lier à des " mondes de production » et à étudier les spécificités des ressources disponibles. Nous identifions ainsi une variété de voies technologiques organisées autour de patrimoines collectifs ayant à la fois une dimension productive et une dimension scientifique.

\section{Keywords:}

green chemistry; paradigm shift; agricultural resources; technological pathways; collective heritage

\begin{abstract}
Green chemistry, a paradigm shift? "Green chemistry" is presented by some authors as the new paradigm for chemistry to meet the challenges of sustainable development. We discuss this hypothesis by focusing on the use of agricultural resources for chemistry (the seventh principle of green chemistry). Following a presentation of the institutional construction around green chemistry, we show that chemistry should be understood as a discipline characterized by goal-oriented learning, in which scientists seek to establish links with "worlds of production" and investigate the specificities of available resources. We identify a variety of technological pathways organized around collective heritages having both productive and scientific dimensions.
\end{abstract}

Les festivités qui ont marqué l'Année internationale de la chimie en 2011 ont oscillé entre célébration de ses apports au bien-être de l'humanité et introspection sur les menaces qu'elle fait peser sur son avenir. Elles ont été l'occasion de mettre en scène les progrès vers une « chimie durable » (Maxim, 2011) réalisés durant les deux dernières décennies, dont une partie importante se réfère à la green chemistry. Cette expression s'est imposée au cours des années 1990, face à des notions concurrentes (benign chemistry, environmental chemistry, chemistry for sustainable development, etc.), grâce à une politique initiée par l'agence américaine de protection environnementale (Environnemental Protection Agency) (Woodhouse et Breyman, 2005 ; Linthorst, 2010). Celle-ci a réussi à mettre en forme un « corps de doctrine » qui s'est traduit par l'édiction de douze principes, connus aujourd'hui de l'essentiel de la communauté des chimistes (Encadré). Des ouvrages d'introduction (Anastas et Warner, 1998 ; Lancaster, 2002 ; Clark, 2002) et des revues dédiées (telles que Green Chemistry en 1998) ont été édités, des

$\overline{\text { Auteurs correspondants }}$ : M. Nieddu, martino.nieddu@univ-reims.fr et F.-D. Vivien, fd.vivien@univ-reims.fr

1 Chercheuse associée au laboratoire REGARDS. 


\section{Encadré : Les douze principes de la chimie verte \\ (Anastas et Warner, 1998, repris par Maxim et Rico-Lattes, 2013, p. 646-647, notre traduction)}

1. Prévention : il vaut mieux produire moins de déchets qu'investir dans l'assainissement ou leur élimination.

2. L'économie d'atomes : les synthèses doivent être conçues dans le but de maximiser l'incorporation des matériaux utilisés au cours du procédé dans le produit final.

3. Lorsque c'est possible, les méthodes de synthèse doivent être conçues pour utiliser et créer des substances faiblement ou non toxiques pour les humains et sans conséquence sur l'environnement.

4. Les produits chimiques doivent être conçus de manière à remplir leur fonction désirée tout en minimisant leur toxicité.

5. Autant que possible, il faut rendre l'utilisation de substances auxiliaires (solvants, agents de séparation, etc.) inutile et, lorsqu' on en utilise, que ce soient des substances inoffensives.

6. Les besoins énergétiques des procédés chimiques doivent être reconnus pour leurs impacts sur l'économie et l'environnement, et doivent être minimisés. Si possible, les réactions de synthèse doivent être conduites dans les conditions de température et de pression ambiantes.

7. Lorsque c'est technologiquement ou économiquement praticable, les matières premières utilisées doivent être renouvelables plutôt que non renouvelables.

8. Lorsque c'est possible, toute déviation inutile du schéma de synthèse (utilisation d'agents bloquants, protection/ déprotection, modification temporaire du procédé physique/chimique) doit être réduite ou éliminée parce que chaque étape requiert des agents additionnels et peut générer des déchets.

9. Les réactifs catalytiques (aussi sélectifs que possible) sont plus efficaces que les réactifs stœchiométriques.

10. Les produits chimiques doivent être conçus de façon à pouvoir se dissocier en produits de dégradation non nocifs à la fin de leur durée d'utilisation, cela dans le but d'éviter leur persistance dans l'environnement.

11. Des méthodologies analytiques doivent être élaborées afin de permettre une surveillance et un contrôle en temps réel et en cours de production avant qu'il y ait apparition de substances dangereuses.

12. Les substances et la forme des substances utilisées dans un procédé chimique devraient être choisies de façon à minimiser les risques d'accident chimique, incluant les rejets, les explosions et les incendies.

instituts chargés de sa diffusion (sur le modèle du Green Chemistry Institute) et des prix célébrant les initiatives dans ce domaine (Presidential Green Chemical Awards aux États-Unis) ont été créés.

Assiste-t-on pour autant à un changement de paradigme, ainsi que l'avancent nombre de chimistes acteurs de la chimie verte et certains économistes ? La notion de paradigme renvoie, chez Kuhn (1983, p. 30), à l'émergence d'une "matrice disciplinaire», fournissant un ensemble cohérent de lois, théories, applications et dispositifs expérimentaux «qui donnent naissance à des traditions particulières de recherche », dont l'assimilation fait d'un étudiant un chercheur appartenant à un groupe scientifique déterminé. Ainsi, la chimie moderne, issue de la révolution des Lumières, serait un ensemble de connaissances dédiées au fractionnement d'éléments naturels en composants élémentaires, à leur purification pour les recombiner afin de créer des molécules ou structures artificielles aux propriétés particulières. Dans ses applications industrielles, elle ne méconnaissait pas sa toxicité potentielle (aucun produit ne peut être considéré comme bénin en soi, pour reprendre une expression chère à Anastas), mais elle se constitue en figure de la modernité, en considérant que les questions de long terme en matière de santé ou d'environnement pouvaient être éludées au nom des avantages tangibles immédiats.
Les économistes évolutionnistes du changement technique élargissent le concept de paradigme en le qualifiant de "technologique", englobant l'ensemble des variables technologiques et économiques entrant dans les innovations ${ }^{2}$. "Dans cette optique, écrivent Oltra et Saint Jean $(2009$, p. 12), le progrès technologique tend à suivre certaines trajectoires technologiques qui correspondent à un compromis évolutif entre les différentes variables, ou dimensions, définies par le paradigme. La dimension environnementale peut donc être vue comme une dimension supplémentaire à intégrer dans ce compromis et dont le poids ne fait qu'augmenter sous les pressions réglementaires. Cette nouvelle dimension tend donc à modifier les trajectoires technologiques, de façon plus ou moins radicale et elle peut, dans certains cas, induire un changement de paradigme technologique. »

L'idée d'une rupture paradigmatique pourrait donc se situer à la fois dans le champ de la science et du laboratoire (l'élaboration de nouvelles stratégies de conception des réactions chimiques en rupture avec le

\footnotetext{
2 "A technological paradigm is defined as a pattern for the solution of selected technoeconomic problems based on highly selected principles derived from the natural sciences. A technological paradigm defines contextually the needs that are meant to be fulfilled, the scientific principles utilised for the task and the material technology to be used » (Dosi, 1988, p. 224-225).
} 
socle de la chimie moderne) et dans le champ technicoéconomique (un renouvellement profond des façons de penser les compromis sur les modes de production et les produits pour atteindre des objectifs de soutenabilité). Ce questionnement, commun à des économistes de l'université de Reims et des chimistes d'unités de recherche de l'Inra et du CNRS, a été mené dans le cadre d'un projet de recherche financé par l'ANR « Chimie et procédés pour le développement durable $»^{3}$. Dans un premier temps, nous allons présenter notre approche transdisciplinaire. Dans un deuxième temps, nous exposerons notre lecture de la littérature qui, dans nos deux disciplines, considère la chimie verte comme un nouveau paradigme. Dans un troisième temps, nous expliquerons pourquoi nous appréhendons ce que nous appelons la « chimie (doublement) verte » comme une construction institutionnelle associée au développement d'une chimie du végétal soumise à des tensions relatives à sa soutenabilité. Dans un quatrième temps, nous verrons qu'au sein de celle-ci est à l'œuvre, non pas un changement de paradigme, mais un ensemble d'apprentissages orientés et localisés réalisés par quatre grandes communautés économico-scientifiques, porteuses de différentes philosophies de la chimie.

\section{Une démarche transdisciplinaire}

Nous avons souhaité faire un objet transdisciplinaire ${ }^{4}$ de cette interrogation relative à un éventuel changement de paradigme induit par la chimie verte. Comme c'est souvent le cas, le dialogue entre disciplines démarrait cependant sur des malentendus. Les chimistes considéraient comme naturel d'interroger les économistes sur l'évaluation de la viabilité économique de solutions émer-

\footnotetext{
3 Nous remercions B. Kurek, directeur de l'UMR Inra Fare, pour sa participation à l'animation de cette ANR (ref. ANR09-CP2D-01-01 AEPRC2V) et aux discussions à l'origine de cet article, ainsi que les collègues chimistes et biochimistes qui, à des degrés divers, ont participé au focus group, avec une mention particulière à F. Duchiron, M. Paquot et F. Quignard. Merci aussi à P. Gallezot pour les pistes précieuses ouvertes au cours d'une interview, ainsi que S. Bouquillon et S. Gatard pour avoir accueilli notre observation participante dans le cadre d'un PIR Ingenotech CNRS.

4 Nous préférerons l'expression transdisciplinarité à celle d'interdisciplinarité. Il s'agit en effet plus d'organiser la connaissance dans un domaine complexe ethétérogène à travers une thématique transversale que d'intégrer nos différentes disciplines dans la construction opérationnelle de concepts communs. Que la chimie verte, en tant que pratique, modifie les frontières disciplinaires - car elle nécessite une intégration entre physicochimie, chimie, science des matériaux, sciences de l'ingénieur et biologie, ou entre différentes sous-disciplines de la chimie - est un autre problème sur lequel nous reviendrons plus loin.
}

gentes données. De la même façon, les économistes étaient tentés d'attendre des chimistes qu'ils leur dessinent la carte des sentiers technologiques crédibles au sein de ce nouveau paradigme, notamment ceux susceptibles d'introduire le plus possible d'innovations environnementales. Cette situation, en grande partie circulaire, aurait pu se traduire par une question de recherche teintée de normativité puisqu'elle visait in fine à la découverte de la «technologie gagnante». Cette tentation était d'autant plus forte que la chimie verte, et plus particulièrement celle liée aux usages des ressources renouvelables, repose en grande partie sur une «économie des promesses » (Joly, 2010) du fait de la construction de discours d'anticipation visant à devenir autoréalisateurs.

La confrontation de nos visions et attentes respectives a permis de nous défaire de nos présupposés initiaux : les chimistes ont intégré que l'on se trouvait dans une phase d'exploration des possibles dans laquelle la coordination des acteurs se fait largement « hors prix », à travers la construction de représentations communes du futur qu'il convenait d'expliciter. Les économistes ont alors proposé aux chimistes différentes représentations de la chimie en sciences sociales. Deux aspects de l'épistémologie de la chimie proposée par BensaudeVincent nous ont intéressés. Le premier est le fait que, face aux revendications d'une "purification» de la démarche scientifique, dont les considérants ne seraient qu'internes à la discipline, Bensaude-Vincent (2013, p. 339) propose d'analyser la technoscience «as an alternative ideal-type value-laden, project-targered, and cross disciplinary research ». Le second est que " the epistemology of chemistry can be adequately defined by the phrase knowing through making » (ibid, p. 334). Nous interprétons cette formule, non pas comme l'absence d'une recherche fondamentale, mais, compte tenu de l'extrême plasticité des réactions chimiques, comme la nécessité à laquelle sont confrontés les chercheurs d'orienter leurs apprentissages dans des directions précises pour finaliser et optimiser leurs réactions ${ }^{5}$. Nous avons pu observer ces processus d'apprentissage et de recherche de directions cibles au cours d'une observation participante menée dans le cadre d'un PIR Ingenotech CNRS. Dans sa recherche fondamentale, le chimiste maîtrise une opération ou un ensemble d'opérations unitaires qui présentent un intérêt spécifique (par exemple, la réussite d'une réaction ou l'amélioration significative de son rendement), mais celles-ci ne prennent sens que dans ce que $\mathrm{l}^{\prime}$ on qualifie, à la suite des historiens des techniques, de "processus d'appropriation ${ }^{6}$. Ces opérations unitaires doivent être

\footnotetext{
5 Par exemple, on peut produire de l'éthylène à partir d'éthanol (biosourcé) ou l'inverse (de l'éthanol à partir d'éthylène $\mathrm{d}^{\prime}$ origine fossile). Orienter les apprentissages dans une direction pertinente ne relève donc pas du seul domaine de la science, mais de la finalité technique et économique ou sociale de la réaction.
} 
appropriées par d'autres acteurs pour se combiner à d'autres savoirs scientifiques ou technologiques afin d'acquérir le statut d'un objet complexe porteur d'une utilité particulière, qu'elle soit d'ordre économique ou $q^{\prime}$ 'elle se situe dans la recherche fondamentale.

Pour des économistes et des philosophes des sciences, si la science apparaît comme une force de production centrale et participe directement aux recompositions des relations et des frontières intersectorielles (Nieddu et al., 2010), elle s'intègre dans les stratégies des acteurs économiques qui cherchent à assurer leur reproduction dans un contexte de changement annoncé et de crise de légitimité de la chimie. Il est donc nécessaire d'observer la science en train de se faire, en interaction avec ces jeux d'acteurs (Pestre, 2006). Ayant retenu que la grande variété de sentiers théoriquement possibles fait que la chimie - du moins, du domaine que nous étudions relève de pratiques d'apprentissages orientés, notre programme de recherche a pu alors se déplacer vers l'élaboration d'une « démarche en compréhension » commune aux économistes et aux chimistes. Au-delà du coencadrement interdisciplinaire de trois thèses, dont celle d'E. Garnier (2012), celle-ci s'est construite grâce à trois dispositifs de recherche :

- un recensement de la littérature relative à la chimie verte (publications de l'American Chemistry Society et des grandes revues concernées par l'usage des ressources renouvelables entre 1990 et 2012) ;

- des entretiens menés auprès de scientifiques de l'Inra et du CNRS à l'occasion de manifestations scientifiques (colloques, écoles-chercheurs, etc.) consacrées à la chimie verte et à la bioraffinerie ;

- l'organisation de quatre sessions d'un focus group ${ }^{7}$ réunissant des microbiologistes, physicochimistes et biochimistes visant à établir des cartographies de com-

\footnotetext{
6 Ce concept renvoie au fait que des acteurs observent un objet scientifique ou technologique et l'interprètent pour un usage particulier : (1) les acteurs scientifiques ou économiques susceptibles d'entrer dans un processus d'appropriation doivent être découverts ; (2) ces derniers doivent poser une hypothèse d'intérêt quant aux promesses contenues dans l'objet. La question de l'exploration des possibilités offertes par une réaction unitaire est donc celle de la fondation d'un « intergroupe » pour une appropriation et des apprentissages orientés spécifiques.

7 Ayant des modalités de fonctionnement faiblement codifiées, le focus group est une technique d'entretien de groupe qui permet de collecter des informations sur un sujet ciblé. Organisé sous la forme d'échanges successifs d'arguments par un animateur dont une des tâches est de maintenir le débat à l'intérieur de frontières établies d'avance, le focus group a l'intérêt de favoriser l'expression et la discussion d'opinions controversées. Il permet, en particulier, comme le notent Moreau et al. (2004, p. 383), de tester des idées nouvelles et minoritaires au sein du groupe - ce qui était un des enjeux de notre recherche.
}

munautés scientifiques. Suivant en cela les principes de l'approche narrative (Dumez et Jeunemaître, 2005), celles-ci étaient falsifiables, au sens de Popper, car les scientifiques ont pu y opposer des contre-exemples ou des contre-interprétations.

Cette démarche s'appuie sur une conceptualisation en termes de patrimoines collectifs propre aux économistes rémois (Nieddu, 2007 ; Nieddu et Vivien, 2010 ; Nieddu et al., 2014), qui permet à la fois de désigner le point de départ des apprentissages des diverses communautés scientifiques, en se référant aux savoir-faire et connaissances dont elles disposent, et le point d'arrivée espéré. Le travail des acteurs consiste à se projeter dans des futurs souhaitables, en identifiant les patrimoines de connaissances, de matériels et de savoir-faire qui méritent d'être préservés et ceux qui seront abandonnés. Le patrimoine est donc, comme le souligne Godard (1990, p. 232), non seulement un héritage transmis, mais une tentative de prise de pouvoir sur le futur, en créant de l'irrévocable. Concrètement, des communautés scientifiques se positionnent en termes d'apprentissages sur la séquence " fractionnement/purification/recombinaison » du végétal de différentes façons, à partir de leurs compétences propres, tout en cherchant des alliances avec les acteurs économiques susceptibles de contribuer à la mise en valeur de leurs programmes de recherche.

\section{Des notions de paradigme aux mobilisations pragmatiques des douze principes}

L'affirmation de l'émergence d'un paradigme scientifique de la chimie verte suppose la remise en cause de l'idée d'un cœur unique du paradigme. Les approches économiques du paradigme, quant à elles, reviennent à poser comme condition de son existence la présence d'innovations radicales. Or, nous allons voir que la construction même des douze principes et leur utilisation dans les communautés scientifiques ou technicoéconomiques font que ces deux conditions - cohérence globale et innovation radicale - ne sont pas nécessaires en chimie verte.

\section{Un changement de paradigme scientifique ? L'affirmation de quatre grandes ruptures}

Nous avons retracé la façon dont l'idée de changement de paradigme est présentée par P. Anastas. Considéré comme le père de la chimie verte, il a été moteur au sein de l'EPA et de l'Américan Society of Chemistry de l'élaboration des douze principes au cours des années 1990, avant d'être nommé responsable de l'EPA's Office of 
Research and Development par Obama en 2010. Dans ses textes, le changement de paradigme désigne parfois la green chemistry dans son ensemble. Par exemple, Anastas et Williamson (1996, p. 2, souligné par nous) écrivent: «This volume details how chemists from all over the world are using their creativity and innovation to develop new synthetic methods, reaction conditions, analytical tools, catalysts and processes under the new paradigm of Green Chemistry. » Plus récemment, on retrouve la même perspective quand Anastas (2012, p. 584-585) présente la réforme de la recherche à l'EPA comme le passage à un nouveau paradigme destiné à aligner la recherche avec les questions de soutenabilité, un article dans lequel il présente la green chemistry comme emblématique de ce changement.

Mais, le plus souvent, Anastas présente la green chemistry comme un assemblage de paradigmes, tels que l'économie d'atomes ou la substitution de solvants. Anastas et Williamson (1996, p. 2) notent ainsi : «It is true that the principle of Atom Economy does not directly address the issue of hazard or toxicity. However, the incorporation of the goals of this paradigm is and needs to be one of the central tenants for any synthesis that is striving toward Green Chemistry. " De la même façon, dans un article pour Science, le mot paradigme se réfère aux changements de problématique autour d'un des fondements de la chimie, l'usage des solvants : " The most elegant way to avoid problems with solvents is not to use them, an approach that has been widely exploited in the paints and coatings industries. Recently, Raston et al. described organic reactions that can be carried out merely by grinding the reagents together with a catalyst, a process that is both cleaner and quicker than the conventional reactions. The experiment may not be easy to scale up to an industrial scale, but it has nevertheless caused a paradigm shift in the synthesis of a whole class of compounds that were previously tedious or difficult to make » (Poliakoff et al., 2002, p. 809).

$C^{\prime}$ est cette référence à un nouveau corpus formé par un assemblage entre différents paradigmes, lequel a précédé la mise en forme des douze principes, qui nous semble constituer le cœur identitaire de la chimie verte. L'originalité de sa revendication comme paradigme tient alors, d'un point de vue épistémologique, à cet assemblage de quatre grandes ruptures dans la philosophie de la chimie et non à l'émergence d'un cœur disciplinaire au sens traditionnel - un point de vue confirmé par les praticiens qui insistent sur l'impossibilité ontologique d'une chimie verte autre qu'interdisciplinaire (Maxim et RicoLattes, 2013). Ces ruptures ont été discutées une à une au sein de notre focus group, ce qui a conduit à constater qu'aucune d'entre elles ne se suffit en soi.

La première rupture porte sur les quantités de coproduits indésirables obtenues lors des opérations de fractionnement-purification. Une nouvelle approche méthodologique, synthétisée dans une formule « d'économie d'atomes" qui fournit une métrique à cette démarche, a été théorisée dès le début des années 1980 (Trost, 1991). Elle cherche à maximiser le nombre d'atomes de réactifs transformés en produit au cours de la synthèse et réduire ainsi la quantité de résidus. Plutôt que de rompre les liaisons chimiques, cela suppose de procéder par réarrangement de la structure du substrat de base. Néanmoins, il nous a été possible - comme le montre également Lancaster (2002) - de trouver des exemples de publications en chimie verte, présentant des réalisations d'économies d'atomes dans le process de production d'un produit hautement toxique, et donc d'améliorer les performances économiques, sans que ne soit remise en cause cette production elle-même.

La deuxième rupture porte sur la maîtrise de l'efficacité des réactions. Aujourd'hui, la catalyse est considérée par certains comme le principe de chimie verte par excellence, car elle permet une plus grande sélectivité (moins de déchets) et une vitesse de réaction plus importante (moins de consommation d'énergie). Toutefois, il ne s'agit ni d'un principe nouveau ni d'un principe spécifique à la chimie verte, puisque tous les grands progrès de la chimie au $X X^{\mathrm{e}}$ siècle (la synthèse de l'ammoniac et de l'acide sulfurique, le craquage du pétrole et la chimie des polymères) sont fondés sur ceux de la catalyse. Les grandes familles de polymères (issus du polyéthylène, polypropylène, polybutadiène) apparaissent grâce à la catalyse (ex : Ziegler - Natta). Il est donc assez curieux de la présenter comme fondatrice d'un nouveau paradigme alors qu'elle est au cœur du métier des chimistes traditionnels depuis presque une centaine d'années.

La troisième rupture porte sur l'utilisation de réactifs et de solvants faiblement ou non toxiques. Beaucoup de solvants traditionnels (les composés organiques volatils) ayant un impact environnemental élevé, il y a un enjeu important dans cette modification des pratiques. Les espérances technologiques ont porté au cours des années 2000 sur de nouveaux solvants, tels que les liquides ioniques qui ont à l'origine été présentés comme les solvants verts par excellence - promesse en grande partie exagérée. La solution de réactions sans solvants, qui représenterait une rupture paradigmatique importante si elle était généralisée, est également explorée.

Dernière rupture, la chimie verte suppose que, dès la conception des produits, soit intégrée la question de la toxicité potentielle (safer by design) des molécules nouvellement produites et de leur caractère persistant dans la nature. C'est elle qui fonde probablement le plus fortement une nouvelle façon de poser la question disciplinaire, en mettant en avant la nécessité d'une approche intégrée et transdisciplinaire, car elle impose une collaboration du chimiste avec d'autres disciplines, 
notamment la biologie, et une révision profonde des bases de sa formation.

\section{Innovations radicales, paradigme technicoéconomique et chimie verte}

L'approche des économistes en termes de paradigme s'inscrit dans une perspective évolutionniste du changement technologique (Nill et Kemp, 2009 ; Oltra et Saint Jean, $\left.2009^{8}\right)$. Elle a été présentée aux chimistes du groupe de recherche comme une analyse de trajectoires technologiques enchâssées dans des paradigmes technologiques dominants et de dynamiques d'innovations notamment environnementales - dans un contexte de pressions réglementaires. Les trajectoires technologiques existantes génèrent de l'inertie, voire des phénomènes de verrouillage technologique, d'où la distinction que ce courant introduit entre différents types d'innovations environnementales, selon qu'elles se situent dans un paradigme ou en rupture avec celui-ci. Ainsi, à la fin des années 1990, Faucheux et Nicolaï (1998, p. 132) notaient que « [1]a plupart des technologies environnementales [...] appartiennent à la catégorie des innovations incrémentales en ce sens qu'elles n'apportent que des perfectionnements aux produits ou aux techniques de production de manière continue au fil du temps. Peu d'entre elles relèvent des innovations radicales qui constituent des ruptures dans l'évolution des procédés ou des produits suscitant une transformation des méthodes de production ou de commercialisation ». Ces auteurs pointaient alors l'existence $\mathrm{d}^{\prime}$ « innovations radicales répondant à des objectifs environnementaux », en citant « la chimie sans chlore, les biocarburants, l'énergie photovoltaïque »... Dix ans plus tard, Oltra et Saint Jean $(2009$, p. 12) retiennent ce cadre analytique et désignent la chimie verte comme l'une de ces innovations de rupture, fondatrices d'un nouveau paradigme.

Caractériser ainsi, de manière normative, la chimie verte par l'existence de technologies environnementales radicales est problématique à plus d'un titre. Cela oblige d'abord à pousser à l'extrême l'identification entre innovations radicales et chimie verte par le biais d'une lecture maximaliste de certains de ses principes, par exemple, le septième, pour justifier du caractère $\mathrm{d}^{\prime}$ 'innovation environnementale radicale des biocarburants. Ainsi, comme l'illustre leur étude du secteur des peintures ${ }^{9}, c^{\prime}$ est l'absence totale de solvant qui caracté-

\footnotetext{
8 V. Oltra et M. Saint Jean ont été invitées dans notre focus group. Dans le cadre d'une collaboration avec elles, une étude bibliométrique a été réalisée, faisant apparaître une communauté " chimie verte » traversée de thématiques dominantes, telles que les liquides ioniques ou la catalyse (Epicoco et al., 2012).
}

rise, selon Oltra et Saint Jean, la chimie verte, alors même que son cinquième principe laisse ouvertes les solutions possibles, dont la simple réduction de l'usage de ces solvants. Ensuite, comme le note Kemp (2010), il est hasardeux de qualifier ex ante une technologie d'environnementale. L'assertion selon laquelle certaines innovations apportent intrinsèquement un bénéfice environnemental doit être discutée à la lumière d'une réflexion sur les éventuels effets rebonds des technologies. Enfin, comme le remarquent Oltra et Saint Jean, ce ne sont peut-être pas les dimensions environnementales des technologies qui expliquent leur adoption et diffusion. Dans le cas des peintures, celles-ci ont été permises par l'amélioration des performances techniques à l'étirement, au séchage ou dans leur aspect et par un rapport coût/bénéfice intéressant en raison de leur rendement élevé... au point où les chimistes de notre groupe se sont demandé si la première explication de leur diffusion ne devait pas être trouvée largement hors de l'argument «chimie verte», alors même que celui-ci est utilisé dans la communication des laboratoires ou des firmes...

\section{Des applications souples et variables des douze principes plutôt qu'une rupture paradigmatique ?}

Les douze principes qui ont rendu célèbre la chimie verte introduisent-ils réellement une rupture paradigmatique, que ce soit d'un point de vue scientifique ou technicoéconomique? Notre reconstitution des raisons de la rapidité de la diffusion du concept de "chimie verte » nous amène à l'expliquer par la souplesse d'exécution obtenue par les grandes firmes nord-américaines, face aux dispositifs réglementaires contraignants du début des années 1980 auxquels elles s'opposaient. Celles-ci reprochaient au régulateur de pousser à des innovations " en bout de chaîne ", gérant « à la sortie » les pollutions générées par le système productif. Elles se montraient en revanche plus favorables à l'adoption de dispositifs institutionnels non contraignants (Woodhouse et Breyman, 2005). L'EPA va donc proposer de travailler sur des démarches innovantes permettant d'agir à long terme sur le design de nouveaux process et produits pour allier des gains économiques et environnementaux. Les douze principes visent alors à intégrer les quatre ruptures présentées plus haut. Mais, s'il y a bien une

\footnotetext{
9 Les principales technologies de réduction d'émission de COV, écrivent Oltra et Saint Jean (2009, p. 15), se situent « dans le paradigme dominant (celui de la pétrochimie), ce qui leur permet de réduire à moindre coût les émissions de COV tout en demeurant compatibles avec les techniques d'application classiques [...]. Les peintures en poudre et les peintures photoréticulables [...] sont les seules totalement sans solvant et pratiquement sans émission de COV. C'est pourquoi nous pouvons dire qu'elles sont les seules technologies s'inscrivant dans le paradigme de la «chimie verte» [...], ce qui représente un saut technologique radical dans le domaine des peintures. »
} 
logique systémique à l'origine de leur édiction, ils se déclinent finalement comme autant de «Tables de la loi » à respecter à des degrés divers. Comme les « Dix commandements ", il y a ceux que l'on tente de suivre (le « tu ne tueras point », sur lequel la chimie est particulièrement attendue et surveillée) et ceux avec lesquels on trouve des accommodements. Chacun peut donc " entrer en green chemistry » à partir de ce guide de bonne conduite en usant des quelques principes qu'il juge atteignables dans le cadre de son travail.

Les interviews réalisées indiquent que les chercheurs se montrent agacés par le succès de ce « concept mou » et très critiques vis-à-vis de ceux d'entre eux ayant tendance à considérer qu'ils font de la chimie verte lorsqu'ils réussissent « à mobiliser au moins plus d'un principe simultanément », pour reprendre l'expression caustique d'un chercheur. Ces chimistes s'interrogent également sur l'articulation entre la chimie verte et le règlement européen REACH qui prend son essor au début des années 2000 (Jouzel et Lascoumes, 2011). Alors que celleci vise à interdire de manière formelle un certain nombre de substances, la chimie verte apparaît essentiellement comme un « dispositif institutionnel cognitif », une façon d'aider le chercheur à penser la question environnementale dans son travail quotidien, tout en laissant à l'industriel la possibilité de choisir les résultats apportant une valeur ajoutée monétaire.

On notera que le champ de la chimie verte se structure autour de revues et de projets de recherche dédiés au développement d'un des principes - par exemple, la catalyse, la mesure de la toxicité ou, pour ce qui va nous intéresser plus particulièrement, l'usage des agroressources. Et, si l'on considère un principe - le cinquième, par exemple, sur les solvants -, il ne nous semble pas possible de faire le tri du point de vue des principes de chimie verte parmi les différents dispositifs entre ceux qui retiennent leur réduction par le biais d'innovations incrémentales et ceux qui proposent des innovations de rupture (chimie sans solvants).

\section{La construction institutionnelle d'une chimie (doublement) verte}

La mobilisation des agroressources peut-elle être vue comme un effet du développement du paradigme de la chimie verte et de l'efficacité de l'édiction du septième principe? L'usage du végétal doit en fait d'abord être rattaché aux stratégies des agro-industries qui, depuis toujours, ont consacré une part de leurs productions à la chimie (Finlay, 2004). Trois phénomènes contemporains les ont conduits à accentuer cette relation (Nieddu et al., 2010 ; Garnier, 2012). Le premier est la survenue d'excédents agroalimentaires dans les grandes nations agricoles qui les amènent à systématiser la réflexion sur les débouchés non alimentaires, à partir du milieu des années 1980. Le deuxième est la perspective du peak oil qui stimule le programme de substitution des carburants liquides d'origine fossile par des carburants biosourcés, sur lequel les acteurs des agro-industries ont commencé à se mobiliser après le premier choc pétrolier et fait les premiers investissements significatifs à partir des années 1990. Le troisième, plus récent, peut-être le seul qui doive être corrélé directement aux enjeux environnementaux de la chimie verte, est lié à la réduction des émissions de $\mathrm{CO}_{2}$, après l'entrée en vigueur du protocole de Kyoto. Craignant une extension des mesures sanctionnant les produits responsables de ces émissions, les entreprises fortement consommatrices de plastiques stimulent un programme de recherche visant à substituer terme à terme, dans les produits issus de la chimie, le carbone d'origine fossile par du carbone d'origine renouvelable, afin de pouvoir exhiber rapidement un bilan carbone plus favorable.

Notre traitement de la revue de littérature montre qu'au début des années 2000 s'y réalise un basculement en faveur des publications consacrées à la chimie du végétal : le poids de la question générique de la chimie verte - la toxicité de la chimie - se trouve relativisé dans les revues de référence de chimie verte et les Presidential Green Chemistry Challenge Awards, tandis que les travaux sur les agroressources y occupent une place toujours plus importante (Garnier, 2012). Le renversement de perspective est saisissant : l'agriculture était le plus souvent traitée dans la littérature de chimie verte des années 1990 comme une industrie polluante, utilisant des molécules toxiques persistantes, et fortement dépendante des produits fossiles. Ce tournant est justifié par les scientifiques par le fait qu'il n'existe pas d'alternative à la chimie du carbone. Le carbone d'origine renouvelable (appelé «carbone bio » par certains) est donc la seule solution pour remplacer le carbone fossile.

C'est dans cette perspective que nous avons revisité les exercices de prospective qui ont été menés, des deux côtés de l'Atlantique, pour se doter du concept de « bioraffinerie » (Christian et al., 2007). Ces actions codifient une stratégie générale fondée sur une heuristique de la chimie, dont Gallezot (2012) note qu'elle est proche de la traditionnelle stratégie de synthèse multiétape fondée sur les hydrocarbures ${ }^{10}$. Elle reprend le schéma de la

\footnotetext{
10 Point de vue confirmé par celui des exercices supportés par la Commission européenne: "L'utilisation de la biomasse comme ressource renouvelable durable est la seule façon de remplacer les sources fossiles par du carbone dans la fabrication de produits chimiques, matériaux et carburants. Pour rendre cette voie compétitive par rapport à celle des produits obtenus à partir de pétrole, une stratégie de bioraffinerie intégrée a été développée [...]. Cette stratégie applique l'approche utilisée dans les raffineries pétrolières à la biomasse [...] pour produire un éventail de produits et maximiser la valeur ajoutée globale » Star-Colibri (2011, p. 10).
} 
division du travail de la raffinerie pétrolière, reposant sur la production de carburants liquides et de cinq grands produits intermédiaires à partir desquels la chimie de base livre ses clients d'aval. La bioraffinerie produirait les carburants liquides équivalents à l'essence et au diesel pétroliers, et un « top 10 » de grands intermédiaires d'origine végétale (Bozell, 2008), qui viendraient se substituer terme à terme aux grandes productions de la chimie de base pétrolière pour servir de briques de base (appelées «molécules plates-formes ») pour le reste de la chimie. Les grands fondements de la chimie moderne - le fractionnement, la purification et la recombinaison - restant au cœur des réactions et process mis en œuvre, il est difficile de plaider pour un changement de paradigme.

Or, il ressort de nos interviews que cette chimie du végétal n'est pas seulement plus difficile, d'un point de vue technicoéconomique ${ }^{11}$, que la chimie du pétrole, elle est peut-être aussi plus « sale », compte tenu des impuretés, des coproduits et de la variabilité de la matière première. Cela conduit certains chercheurs à exprimer leur inquiétude de voir la réarticulation des outils de chimie verte autour $d u$ septième principe négliger $d$ 'autres principes, et produire aussi de nouveaux problèmes environnementaux. La question se déplace donc de la chimie verte vers le problème de la soutenabilité économique de la bioraffinerie (avec des taux de rendement des réactions sur les produits issus de la biomasse encore trop faibles) et les débats sur sa soutenabilité environnementale ou sociale. D'où notre expression de «chimie doublement verte " pour désigner non un nouveau paradigme, mais les tensions autour de la chimie du végétal.

\section{Un exercice d'identification de communautés en chimie doublement verte}

Notre groupe de travail a d'abord pointé la force de la compétition entre deux grandes voies de traitement de la biomasse reconnues dans les exercices de prospective européens et américains, lesquelles reprennent le corps traditionnel de la chimie pétrolière pour en appliquer les grands principes de craquage catalytique et de process multiétapes (accélérés par les catalyses, donc qualifiés de multiétapes catalytiques) de reforming à partir d'éléments purifiés.

La première s'appuie sur des procédés thermochimiques (essentiellement dédiés à la production de

\footnotetext{
11 Elle est plus difficile car une partie importante des réactions de synthèse, qui sont documentées aujourd'hui sur des biomasses, est fondée sur ce qu'on appelle des réactions stœchiométriques, qui atteignent de faibles taux de rendements réactionnels par opposition aux réactions catalytiques que l'industrie pétrolière a énormément explorées pour optimiser ses process.
}

biodiesel et de ses coproduits, mais pouvant également couvrir d'autres productions), issus de patrimoines technologiques bien connus - tel le procédé FischerTropsh, régulièrement revisité depuis les années 1920, les travaux les plus récents portant sur une voie dite BTL (Biomass to Liquids). La seconde relève des patrimoines de procédés biochimiques de fermentation des sucres à travers lesquels sont formés des alcools (l'éthanol) utilisables dans les carburants ou en chimie, eux aussi bien connus des agro-industries. La tentation était forte de conclure à la formation d'un double «dominant design » dans la stratégie de bioraffinerie fondée sur le couple biocarburants/coproduits. Nous disposions cependant de revues de littérature critiques (Gallezot, 2007; Marquardt et al., 2010) et d'exemples n'entrant pas dans ces deux premières voies, sur lesquels nous avons fait réagir le focus group.

Il est apparu dans les discussions que ces autres voies sont ontologiquement différentes de la stratégie de cracking approfondi, relevant des savoirs pétroliers (et que certains cherchent à renouveler par une voie biochimique), parce qu'elles cherchent à préserver et aménager des fonctionnalités contenues dans la matière première renouvelable. Elles renvoient à deux autres grands types de patrimoines de connaissances et de savoir-faire technologiques. Le premier consiste à réaliser l'extraction de macromolécules ou de composés actifs présentant un intérêt particulier et à se livrer à des transformations chimiques limitées à la cible d'utilisation retenue, dans une chimie de fonctionnalités (dont l'oléochimie qui conserve les structures complexes des huiles ou la parfumerie issue de produits naturels peuvent être considérées comme des archétypes). Le second consiste à conserver les grands composants de plantes (amidon, celluloses) pour les fonctionnaliser, en combinant chimie et science des matériaux (amidons thermoplastiques, par exemple).

Nous aboutissons donc à la typologie suivante (Tab.) :

Tableau. Quatre patrimoines productifs (PP) à l'œuvre $(\mathrm{C}=$ nombre d'atomes de carbone constituant la base des opérations des chimistes).

PP1 - Déconstruction thermique radicale en chaînes C1-C5
PP2 - Déconstruction radicale biotechnologique pour des
C2 - C10
PP3 - Extraction de macromolécules contenant un principe
actif C5-C30 (oléochimie, par exemple) et fonctionnalisation
PP4 - Déconstruction limitée aux grands composants
Cx - Cn et fonctionnalisation

Source : extrait de Bliard et Nieddu, 2011.

Ces différences renvoient à l'interrogation du chimiste sur sa façon de concevoir le rapport à la matière première renouvelable. Dans le paradigme moderne, la 
spécificité de celle-ci peut être considérée comme une «non-question", si ce n'est sous l'angle des difficultés supplémentaires que la structure ou la variabilité du vivant entraîne dans la séquence séparation/purification/reforming. Elle peut, au contraire, prendre une place centrale dans la réflexion: Verhée (2010), par exemple, explique qu'il y a un paradoxe à produire du biodiesel à partir des huiles végétales, issues de synthèses complexes réalisées par la nature, formant des chaînes longues de carbone (C6 à Cxx), pouvant être préservées plutôt que détruites car riches en fonctionnalités.

Ces différences apparaissent aussi en rapport avec les interrogations des économistes au sujet d'un changement de paradigme technicoéconomique. La stratégie des voies biochimique ou thermochimique se comprend si l'on cherche, à travers de très grandes unités de chimie de base, à « réutiliser les chaînes de valeurs existantes, le scénario le plus économique à première vue » (Marquardt et al., 2010, p. 2228). Elles convergent dans un modèle unique où « tout doit changer » (les sources de matière première, les circuits de fourniture, les catalyseurs) « pour que rien ne change » à l'aval de la chimie de base. Les trajectoires d'apprentissage doivent donc être replacées dans ce contexte où les catégories d'innovation incrémentale et radicale deviennent fragiles : ce qui est présenté comme innovations radicales (sur les catalyseurs, les nouveaux solvants, etc.) est en effet mis au service de la progression incrémentale de la raffinerie vers la bioraffinerie...

Plutôt qu'une situation évoluant vers un paradigme unifié de la chimie verte, on observe donc un ensemble d'apprentissages localisés réalisés par diverses communautés à l'aide d'héritages de connaissances et de process différents. Notre focus group s'est attaché à étudier la façon dont elles travaillent et mettent en scène leurs efforts visà-vis des principes de chimie verte.

Dans les procédés thermochimiques, qui consomment beaucoup d'énergie, l'accent va être mis sur une hybridation avec les principes de chimie verte qui réside dans la recherche d'une réduction des coûts énergétiques et des étapes de fractionnement catalytique (principe 9), en cherchant à sélectionner des agents de séparation moins nocifs (principe 5). Les communautés, nées des espérances des biotechnologies, espèrent réduire de façon significative ce coût énergétique (principe 6) et obtenir des réactions permettant de maximiser la quantité de matière première terminant dans le produit final (principe 2) grâce aux progrès dans l'ingénierie des enzymes utilisés en catalyse (principe 9).

Les deux autres communautés se réfèrent à d'autres principes: des procédés d'extraction «doux» (principe 3) afin que les principes actifs soient conservés, l'élimination d'étapes de fractionnement avant une chimie de fonctionnalisation (principe 8). Par exemple, la synthèse dite " one-pot », en anglais, est mise en exergue, car la cascade de réactions catalytiques se fait en continu dans une même cuve au lieu de passer par les étapes de séparation-purification, et sans se préoccuper des impuretés, dès lors que celles-ci n'inhibent pas les fonctionnalités recherchées (Gallezot, 2007, p. 295), ce qui conduit à la minimisation des déchets et des coûts énergétiques (principes 1, 2, 6, 8 et 9). Le " benign by design » dès la première étape est mis également en avant dans ces deux héritages productifs avec une conception des produits permettant d'arriver directement au produit biodégradable (principe 10) des biosolvants ou biolubrifiants dans le PP3, ou des amidons modifiés pour des plastiques biodégradables dans le PP4. Cette conception s'appuie sur des procédés de réaménagement des structures tels que la photochimie, la photoréticulation ou l'extrusion réactive qui évitent l'usage de solvants et $\mathrm{d}$ 'auxiliaires de synthèse polluants (principes 1, 2, 6, 8, 9 + principe 5) [Fengwei et al., 2006 ; Evon, 2008].

\section{Conclusion}

L'hypothèse d'un changement de paradigme de la chimie moderne vers une chimie verte, dont il suffirait d'étudier la diffusion et d'identifier les verrous technologiques à lever, s'avère être des plus optimistes. Notre travail transdisciplinaire conduit à l'invalider. Nous n'observons pas - malgré le souhait manifeste de certains de ses fondateurs et de certains économistes - la formation d'une matrice disciplinaire unifiant des façons de pensée et des pratiques. La chimie verte relève plutôt $\mathrm{d}^{\prime}$ un « travail institutionnel » qui s'opère $\mathrm{d}^{\prime}$ abord au sein de la discipline sous la forme d'une édiction de principes non contraignants, laissant ouverte la question du changement de philosophie de la chimie souhaitée par les auteurs du concept. Grâce à ces principes, on peut faire de la chimie verte, tout en faisant comme avant, à savoir améliorer l'efficacité des réactions, valoriser économiquement l'ensemble des fractions de la matière première, faire progresser la catalyse, etc. L'impression de convergence, qui pouvait ressortir des exercices de prospective technologique relatifs aux molécules platesformes, attendues comme substituts aux molécules pétrolières, révèle un travail institutionnel d'un autre type : celui d'acteurs, cherchant, à partir d'une vision du futur fondée sur le mimétisme avec la voie pétrolière, à piloter la recherche actuelle.

De fait, les chimistes font face à un dilemme permanent entre deux philosophies de la chimie dans l'usage de la biomasse ${ }^{12}$. D'une part, les sentiers de cracking

\footnotetext{
12 On reprend ici les éléments de la réponse faite par A. Lattes à une première présentation des différents héritages de patrimoines collectifs en chimie, lors de l'école-chercheurs du CNRS «Chimie pour le développement durable » à Cargèse en 2009.
} 
réduisent la variabilité et la complexité à des intermédiaires de base simples (tels que les diacides) pouvant entrer dans la chaîne de valeur des produits chimiques. D'autre part, les sentiers qui cherchent «à tirer parti de la nature » plutôt que de la briser par un craquage nécessairement coûteux en énergie renvoient à la nécessité de connaissances fondamentales sur les structures de la matière, parfois cruellement absentes. Ce constat nous a amenés à développer un dispositif analytique alternatif à celui de paradigme, en termes de patrimoines collectifs. La chimie a été appréhendée comme une discipline d'apprentissages orientés, dont les scientifiques cherchent à lier leurs connaissances à des «mondes de production » et aux spécificités des ressources disponibles. On a ainsi pu identifier quatre grandes familles selon le type de traitement de la biomasse qu'elles proposent. Chacune de ces voies technologiques repose sur des héritages productifs et de connaissances, irréductibles les uns aux autres, qui sont portés par la permanence d'acteurs économiques et de ressources aux caractéristiques spécifiques. Chacun de ces héritages produit son propre discours sur la chimie verte et sa propre trajectoire d'apprentissage localisé.

\section{Références}

Anastas, P.T., 2012. Fundamental changes to EPA's research enterprise: The path forward, Environmental Science $\mathcal{E}$ Technology, 46, 2, 580-586.

Anastas, P.T., Williamson, T.C., 1996. Green chemistry: An overview, in Anastas, P.T., Williamson, T.C., (Eds), Green Chemistry: Designing for the Environment, ACS Symposium Series 626, Washington D.C., ACS Publications, 1-17.

Anastas, P.T., Warner, J.C., 1998. Green Chemistry, Theory and Practice, Oxford, Oxford University Press.

Bensaude-Vincent, B., 2013. Chemistry as techno-science?, in Llored, J.-P., (Ed.), The Philosophy of Chemistry, Newcastle, Cambridge scholar publishing, 330-342.

Bliard, C., Nieddu, M., 2011. Communities and creation of knowledge as common goods in doubly green chemistry. Communication at The 3rd International Conference on Biodegradable and Biobased Polymers, Strasbourg, 29-31 August.

Bozell, J.J., 2008. Feedstocks for the future: Biorefinery production of chemicals from renewable carbon, CLEAN Soil, Air, Water, 36, 8, 641-647.

Christian, D., Jolly, G., Grenier de March, G., Steinmetz, V., 2007. La bioraffinerie: un concept qui fait son chemin, Biofutur, 282, 20-23.

Clark, J.H., 2002. Introduction, in Clark, J.H., Macquarrie, D.J. (Eds.), Handbook of Green Chemistry and Technology, Oxford, Blackwell, 1-9.

Dosi, G., 1988. The nature of the innovative process, in Dosi, G., Freeman, C., Nelson, R., Silverberg, G., Soete, L. (Eds), Technical Change and Economic Theory, London/New York, Pinter Publishers, 221-238.

Dumez, H., Jeunemaître, A., 2005. La démarche narrative en économie, Revue Économique, 56, 4, 983-1005.
Epicoco, M., Oltra, V., Saint Jean, M., 2012. Mapping the scientific knowledge of the Green Chemistry community, Cahiers $d u$ GREThA, 10, http://cahiersdugretha.ubordeaux4.fr/2012/2012-10.pdf

Evon, P., 2008. Nouveau procédé de bioraffinage du tournesol plante entière par fractionnement thermo-mécano-chimique en extrudeur. Thèse, Université de Toulouse.

Fengwei, X., Long, Y., Hongshen, L., Ling, C., 2006. Starch modification using reactive extrusion, Starch/Stärke, 58, 131-139.

Faucheux, S., Nicolaï, I., 1998. Les firmes face au développement soutenable: changement technologique et gouvernance au sein de la dynamique industrielle, Revue d'Économie Industrielle, 83, 127-146.

Finlay, M.-R., 2004. Old efforts at new uses: A brief history of chemurgy and the American search for biobased materials, Journal of Industrial Ecology, 7, 3-4, 33-46.

Gallezot, P., 2007. Process option for converting renewable feedstocks to bioproducts, Green Chemistry, 9, 295-302.

Gallezot, P., 2012. Critical review: Conversion of biomass to selected chemical products, Chemical Society Reviews, 41, 1538-1558.

Garnier, E., 2012. Une approche socio-économique de l'orientation des projets de recherche en chimie doublement verte. Thèse de doctorat ès Sciences économiques, Université de Reims Champagne Ardenne.

Godard, O., 1990. Environnement, modes de coordination et systèmes de légitimité : analyse de la catégorie de patrimoine naturel, Revue Économique, 2, 215-242.

Joly, P.-B., 2010. On the economics of techno-scientific promises, in Akrich, M., Barthe, Y., Muniesa, F., Mustar, P. (Eds.), Débordements. Mélanges offerts à Michel Callon, Paris, Presses des Mines, 203-222.

Jouzel, J.-N., Lascoumes, P., 2011. Le règlement REACH : une politique européenne de l'incertain. Un détour de régulation pour la gestion des risques chimiques, Politique Européenne, 1, 33, 185-214.

Kemp, R., 2010. Sustainable technologies do not exist!, Ekonomiaz. Revista Vasca de Economia, 75, 2-17.

Kuhn, T.S., 1983. La Structure des révolutions scientifiques, Paris, Flammarion (édition anglaise originale 1962).

Lancaster, M., 2002. Green Chemistry, an Introductory Text, Cambridge, Royal Society of Chemistry.

Linthorst, J.A., 2010. An overview: Origins and development of green chemistry, Foundations of Chemistry, 12, 55-68.

Marquardt, W., Harwardt, A., Voll, A., 2010. The biorenewables opportunity: toward next generation process and product systems, AIChE, 56, 9, 2228-2235.

Maxim, L. (Ed.), 2011. La Chimie durable, au-delà des promesses, Paris, CNRS Éditions.

Maxim, M., Rico-Lattes, I. 2013. Sustainable chemistry, in Llored J.-P. (Ed.), The Philosophy of Chemistry, Newcastle, Cambridge Scholar publishing, 640-658.

Moreau, A., Dedianne, M.C., Letrilliart, L., Le Goaziou, M.F., Labarère, J., Terra, J.L., 2004. S'approprier la méthode du focus group, La Revue du Praticien. Médecine Générale, 18, 645, 382-384.

Nieddu, M., 2007. Le patrimoine comme relation économique, Économie Appliquée, 60, 3, 31-57. 
Nieddu, M., Garnier, E., Bliard, C., 2010. L'émergence d'une chimie doublement verte, Revue d'Économie Industrielle, 132, 4, 53-84.

Nieddu, M., Vivien, F.-D., 2010. Patrimoine, territoire et développement durable, in Zuindeau, B. (Ed.), Développement durable et territoire, Villeneuve-d'Ascq, Presses universitaires du Septentrion, 133-142.

Nieddu, M., Garnier, E., Bliard, C., 2014. Patrimoines productifs collectifs versus exploration/exploitation : le cas de la bioraffinerie, Revue Économique, à paraître (disponible en prépublication sur http://www.cairn.info/revueeconomique-2014-0.htm).

Nill, J., Kemp, R., 2009. Evolutionary approaches for sustainable innovation policies: From niches to paradigm?, Research Policy, 38, 4, 668-680.

Oltra, V., Saint Jean, M., 2009. Innovations environnementales et dynamiques industrielles, Cahiers du GREThA, 22,
Université de Bordeaux IV (online: http://cahiersdugretha. u-bordeaux4.fr/2009/2009-22.pdf).

Pestre, D., 2006. Introduction aux Science Studies, Paris, La Découverte.

Poliakoff, M., Fitzpatrick, J.M., Farren, T.M., Anastas, P.T., 2002. Green chemistry: Science and politics of change, Science, 297, 5582, 807-810.

Star-Colibri, 2011. European Biorefinery Joint Strategic Research Roadmap (online: http://www.star-colibri.eu/files/files/ roadmap-web.pdf).

Trost, B.M., 1991. The Atom Economy: A search for synthetic efficiency, Science, 254, 5037, 1471-1477.

Verhée, R., 2010. Dilemma: Petrochemistry and oleochemistry as resources for fuel and oleochemicals, European Journal of Lipid Science and Technology, 112, 4, 427.

Woodhouse, E.J., Breyman, S., 2005. Green chemistry as social movement?, Technology \& Human Values, 30, 2, 199-222.

Reçu le 13 novembre 2012. Accepté le 5 décembre 2013. 